Research Article

\title{
Stopping Power Modulation by Pump Waves of Charged Particles Moving above Two-Dimensional Electron Gases
}

\author{
Yahong Yang $\mathbb{D}^{1},{ }^{1}$ Ya Zhang $\mathbb{D}^{2},{ }^{2}$ Lin $Y i,{ }^{1}$ and Wei Jiang $\mathbb{D D}^{1}$ \\ ${ }^{1}$ School of Physics, Huazhong University of Science and Technology, Wuhan 430074, China \\ ${ }^{2}$ Department of Physics, Wuhan University of Technology, Wuhan 430070, China \\ Correspondence should be addressed to Ya Zhang; yazhang@whut.edu.cn and Wei Jiang; weijiang@hust.edu.cn
}

Received 7 January 2021; Revised 9 March 2021; Accepted 13 March 2021; Published 30 March 2021

Academic Editor: Dieter H.H. Hoffmann

Copyright (c) 2021 Yahong Yang et al. This is an open access article distributed under the Creative Commons Attribution License, which permits unrestricted use, distribution, and reproduction in any medium, provided the original work is properly cited.

The perturbation electron density and stopping power caused by the movement of charged particles above two-dimensional quantum electron gases (2DQEG) have been studied in numerous works using the quantum hydrodynamic (QHD) theory. In this paper, the QHD is modified by introducing the two-dimensional electron exchange-correlation potential at high density $V_{x c 2 \mathrm{DH}}$ and the pump wave modulations. Based on the modified QHD, the perturbation electron density and stopping power are calculated for pump waves with various parameters. The results show that the stopping power values are more accurate after considering $V_{x c 2 \mathrm{DH}}$. Under the modulation of pump waves with the wavelength from $0.1 \mathrm{~nm}$ to $0.1 \mathrm{~cm}$, the perturbation electron density of 2DQEG and the stopping power of charged particles show periodic changes. Under the modulation of pump waves with $\lambda=1.76 \times 10^{-4} \mathrm{~cm}$ and $\Phi_{0}=2 \times 10^{10} e / \lambda_{f}$, the average stopping power with respect to the time phase $\theta$ becomes negative, which means that the charged particles will gain energy and can be accelerated. This is a new phenomenon in the fields of 2DQEG and of great significance in surface physics and surface modification in nanoelectronic devices with beam matter interactions.

\section{Introduction}

Accompanied by the two-dimensional quantum electron gases (2DQEG) is confirmed to exist on the surface of the metals and semiconductor heterostructures. The interaction between charged particles and plasma targets has attracted a lot of attention in surface physics [1-6]. The incident particles can also be used as probes to detect the properties of 2DQEG, or as a powerful tool of surface character modification.

In the study of the interaction between charged particles and plasma targets, people are particularly interested in the analytical calculation of energy loss [7] and the density distribution of the plasma targets [8]. Stopping power is used to describe the energy loss per unit length [6,9-15]. As an important physical quantity to study the interaction between charged particles and the plasma targets, a lot of experiments and theoretical studies have been done in the past decade [16-28]. The stopping power of charged particles can be modulated by the incident velocity of charged particles [5], the amount of charge of charged particles, the density of plasma targets [5], varying altitude parameter between charged particles and 2DQEG $[4,5,29]$, the external magnetic field [30,31], the spin effects [32], and so on.

In order to understand the interaction between charged particles and plasma targets in the presence of a laser field, a lot of theoretical studies have been carried out [33-39]. Within the dielectric formalism, Arist et al. [33] studied the influence of a strong laser field on the stopping power for charged particles in low-density nondegenerate plasmas, and the expression of stopping power is given when a laser field exists. The results show that the laser field reduces the stopping power of plasma and makes it anisotropic through the polarization of the electric field wave. Within the random-phase approximation theory, Nersisyan [34] also investigated the stopping and acceleration effect of protons in a plasma in the presence of an intense radiation field and calculated the stopping power of charged particles in the high-frequency limit. With two-dimensional electrostatic particle-in-cell (2DPIC) simulation, $\mathrm{Hu}$ [35] studied energy 
loss of ion beam when plasma irradiated by a strong laser pulse and the dynamic polarization of plasma. Pertaining results make it clear that stopping power gets strongly modulated by the laser field.

However, these research methods are no longer applicable when studying the stopping power modulation by pump waves of charged particles moving above the 2DQEG. In the study of Nersisyan et al., when considering the interaction between laser and three-dimensional plasma, the expression of the vector potential $A$ of electromagnetic waves was used [34]. However, the vector potential expression of the electromagnetic wave $A$ cannot be written for the interaction of 2DQEG and laser. Another feasible method to implement electromagnetic waves is to ignore the effect of the magnetic field and write down the expression for the electric field term of electromagnetic waves [40]. The 2DPIC method cannot be used either to study the interaction between charged particles and 2DQEG. Because the charged particles are above the 2DQEG, a three-dimensional electrostatic particle-in-cell (3DPIC) method is required. However, the 3DPIC method is currently difficult to implement due to a large amount of calculation [35].

In this paper, a modified linear QHD is used to study the modulation effect of pump waves on the perturbation electron density and stopping power caused by the movement of charged particles above the 2DQEG. The outline of this article is as follows. In Section 2, the modified QHD is introduced to include the laser pump wave modulation and the two-dimensional electron exchange-correlation potential of high-density $V_{x c 2 \mathrm{DH}}$ effects. The general expressions of perturbation electron density of 2DQEG and stopping power of incident charged particles are derived by the modified QHD theory. In Section 3, the numerical results of perturbation electron density of 2DQEG and stopping power of incident charged particles modulated by pump waves with various physical parameters (wavelength $\lambda$, amplitude $\Phi_{0}$, and time phase $\theta$ ) are given. Section 4 gives a summary. Gauss system of units is adopted in this paper.

\section{Quantum Hydrodynamic Model}

2.1. Physic Model. The schematic diagram is given in Figure 1. 2DQEG is an idealized model, which corresponds to the electronic components on the surface or interface of metal or semiconductor in a very thin layer (only a few layers of atoms' thickness). For the convenience of studying, it is generally considered that the thickness of such twodimensional electron gas is taken negligible [41]. Consider an infinite 2DQEG composed of free electrons and motionless ions located on the plane $z=0$, and use a Cartesian coordinate system to represent its position in the region $z>0$ and $z<0 . n_{0}$ is the equilibrium density of electrons and ions. At equilibrium, 2DQEG density satisfies the relation $n_{i}=n_{e}=n_{0}$ [32]. Considering that the quantum effect is more significant in the high-density electron gas, we take the equilibrium density of electrons and ions as $n_{0}=5.69 \times 10^{15} \mathrm{~cm}^{-2}$, which is the density of aluminum surface [5].

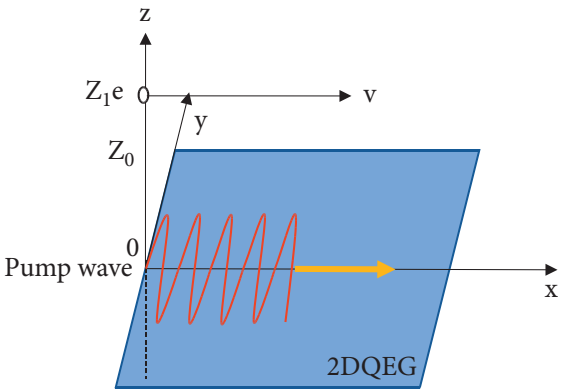

FIgURE 1: The schematic diagram of the interaction between pump waves, charged particles, and 2DQEG.

Charged particles move in the $x$ direction parallel to the 2DQEG plane, with a velocity of $v=2 v_{B}$ and a height of $z_{0}=9 \lambda_{f}$ away from the 2DQEG. Here, $v_{B}=e^{2} / \hbar$ is Bohr velocity, $\lambda_{f}=1 / \sqrt{2 \pi n_{o}}=5.29 \times 10^{-9} \mathrm{~cm}$ is Fermi wavelength, and $e$ is the elementary charge. The density of charged particles with charge $Z_{1} e$ can be expressed as $\rho_{\text {ext }}=Z_{1} e \delta(\mathbf{r}-\mathbf{v} t) \delta\left(z-z_{0}\right)$, where $\mathbf{r}=\mathbf{r}(x, y)$.

We assume that the pump waves are collimated, monochromatic, continuous electromagnetic waves with wavelength $\lambda$ propagating in the $+x$ direction. Along the $+x$ direction, the pump wave with the wavelength from $0.1 \mathrm{~nm}$ ( $\gamma$ rays) to $0.1 \mathrm{~cm}$ (microwave) irradiates the 2DQEG. The electric field component of the pump wave acts on the 2DQEG as an external high-frequency electric field in the plane of $x 0 y$, and the reaction of the 2DQEG on the pump wave is without the scope of our consideration [35, 42]. The electrons in the 2DQEG are affected by the pump wave electric field, which changes the spatial distribution of electrons in the 2DQEG. Then, the exciting electric field by electrons in 2DQEG acts on the incident particle, which affects the velocity of the incident particle, thus affecting the stopping power of incident particles. In terms of electric field, the pump waves can be expressed as $\mathbf{E}=-i \mathbf{k}_{0} \Phi_{0} \exp \left[-i\left(\omega_{0} t-k_{0} x\right)\right]$, where $\mathbf{k}_{0}$ is the wave vector of pump waves [40]. The time variable of the pump wave is $t$. The wavelength of the pump wave is $\lambda=1 / k_{0} . \omega_{0}$ is the frequency of the pump wave, where $\omega_{0}=k_{0} c$, in which $c=$ $2.998 \times 10^{10}$ is the speed of light. The intensity of the pump wave can be calculated by $W_{p}=c\left(k_{0} \Phi_{0}\right)^{2} / 8 \pi$ [34], and the maximum intensity of the pump wave is $10^{23} \mathrm{~W} \mathrm{~cm}^{-2}$ in this paper. When $k_{0}$ is constant, the strength of the pump wave is varying with the pump amplitude $\Phi_{0}$.

2.2. Mathematical Model. As a powerful research method, QHD theory is widely used in plasma research [43-45]. Li et al. $[4,5,46]$ conducted a series of studies on the stopping power of charged particles above the 2DQEG using linearized QHD theory, which is consistent with dielectric response theory and experimental results. In our research, the equilibrium electron density of $2 \mathrm{DQEG}$ will be disturbed by pump waves and injecting charged particles, generating charged fluid velocity field $\mathbf{u}_{e}(\mathbf{r}, t)$ and electron gas density $n_{e}(\mathbf{r}, t)$. According to the linearized QHD theory, the density 
and velocity of electrons on the 2DQEG surface can be described by the continuity equation

$$
\frac{\partial n_{e}}{\partial t}+\nabla_{\|} \cdot\left(n_{e} u_{e}\right)=0
$$

the momentum-balance equation

$$
\begin{aligned}
m_{e}\left(\frac{\partial \mathbf{u}_{e}}{\partial t}+\mathbf{u}_{e} \cdot \nabla_{\|} \mathbf{u}_{e}\right)= & \left.e \nabla_{\|} \Phi\right|_{z=0}-\nabla_{\|} w_{e} \\
& +\frac{\hbar^{2}}{2 m_{e}} \nabla_{\|}\left(\frac{1}{\sqrt{n_{e}}} \nabla_{\|}^{2} \sqrt{n_{e}}\right)-\gamma m_{e} \mathbf{u}_{e} \\
& -e \mathbf{E}-V_{x c 2 \mathrm{DH}} \frac{\nabla n_{e 1}}{n_{e}},
\end{aligned}
$$

and Poisson's equation

$$
\nabla^{2} \Phi=4 \pi e\left[n_{e} \delta(z)-n_{0} \delta(z)-Z_{1} \delta(\mathbf{r}-\mathbf{v} t) \delta\left(z-z_{0}\right)\right] .
$$

Here, $\nabla_{\|}=\partial / \partial x \mathbf{e}_{x}+\partial / \partial y \mathbf{e}_{y}$, whereas in equation (3), the differentiation $\nabla=\partial / \partial x \mathbf{e}_{x}+\partial / \partial y \mathbf{e}_{y}+\partial / \partial z \mathbf{e}_{z}$ is unrestricted. $\Phi$ denotes total electrostatic potential built on wake potential $\Phi_{\text {ind }}$ caused by the collective excitation of electron gas and the charged particle's external potential. On the right-hand side of equation (2), the first term is the force of electrons on the 2DQEG surface due to the tangential electric field, and the second term is the force caused by the quantum statistical effect. Here, $w_{e}=\pi \hbar^{2} n_{e} / m_{e}$ refers to the quantum statistical pressure term acting on electrons in 2DQEG, the third term is the force due to the quantum diffraction effects, the fourth term is the frictional force caused by the interaction between electrons and positive charge background where $\gamma$ is the friction coefficient, the fifth term is the force on electrons owing to the electric field of pump wave, and the last term is the force on electrons due to electron exchange-correlation potential $V_{x c 2 \mathrm{DH}}$. The validity of QHD equations (1) and (2) implies that the off-diagonal components of the pressure tensor and viscosity remain negligible.

2.3. Exchange-Correlation Potential. Exchange-correlation potential is an important quantum effect term. Mir et al. considered the exchange-correlation potential when studying degenerate plasmas [40]. Haas derived a quantum fluid equation containing exchange correlation, which is described by an effective potential [47]. Pollack nd Perdew have made an in-depth study on the exchange-correlation potential of two-dimensional electron gas [48]. However, the exchange-correlation potential is not considered in the study of the interaction between incident particles and 2DQEG using the quantum hydrodynamic method so far. The three-dimensional electron exchange-correlation potential is expressed as follows:

$$
\begin{aligned}
V_{x c 3 D^{\prime}}= & \frac{0.985}{3} \frac{e^{2}}{\varepsilon}\left(n_{0}\right)^{1 / 3}+\frac{0.985 \times 0.034}{3 a_{B}} \frac{e^{2}}{\varepsilon} \\
& \cdot \frac{18.37 a_{B}\left(n_{0}\right)^{1 / 3}}{1+18.37 a_{B}\left(n_{0}\right)^{1 / 3}},
\end{aligned}
$$

where $a_{B}=\varepsilon \hbar^{2} / m_{e} e^{2}$ is effective Bohr atomic radius, in which $\varepsilon=1$ is the relative dielectric constant and $m_{e}$ is the electron mass [40]. We adapt the dimension of the three-dimensional electron exchange-correlation potential to the dimension of the two-dimensional electron exchange-correlation potential based on [40].

$$
V_{x c 3 D}=\frac{0.985}{3} \frac{1}{r_{s}(2 \pi)^{1 / 2}}+\frac{0.985 \times 0.034}{3} \frac{18.37 / r_{s}(2 \pi)^{1 / 2}}{1+18.37 / r_{s}(2 \pi)^{1 / 2}} .
$$

The two-dimensional electron exchange-correlation potential of low density is then expressed as follows [40]:

$$
V_{x c 2 \mathrm{DL}}=0.5058\left[\frac{1.3311}{r_{s}^{2}}\left(\sqrt{1+1.5026 r_{s}}-1\right)-\frac{1}{r_{s}}\right] \text {, }
$$

and the two-dimensional electron exchange-correlation potential of high density is expressed as follows [40]:

$$
V_{x c 2 \mathrm{DH}}=\frac{-0.6002}{r_{s}}
$$

where $r_{s}=\left(2 \pi n_{0} a_{B}^{2}\right)^{-1 / 2}$ is the Wigner-Seitz radius associated with the 2DQEG density [48].

The $V_{x c 3 D}$ (solid line), the $V_{x c 2 \mathrm{DL}}$ (dashed line), and the $V_{x c 2 \mathrm{DH}}$ (dotted line) are shown in Figure 2 . Here, the value of $V_{x c 3 D}$ is positive, and the values of $V_{x c 2 \mathrm{DL}}$ and $V_{x c 2 \mathrm{DH}}$ are negative. It is reasonable to assume that two-dimensional electron exchange-correlation potential has completely different properties from its three-dimensional electron homologue. The Wigner-Seitz radius of $2 \mathrm{DQEG}$ that we are considering is $r_{s}=1$, the corresponding density is $n_{0}=5.69 \times 10^{15} \mathrm{~cm}^{-2}$, and the corresponding exchangecorrelation potentials are $V_{x c 3 D}=0.1362, V_{x c 2 \mathrm{DL}}=$ -0.114 , and $V_{x c 2 \mathrm{DH}}=-0.6002$ (these are dimensionless values). In previous studies of the interaction between charged particles and 2DQEG, the exchange-correlation potential was ignored due to its small value. Here, the absolute value of $V_{x c 2 \mathrm{DH}}$ is the largest compared with $V_{x c 3 D}$ and $V_{x c 2 \mathrm{DL}}$ and the absolute value of $V_{x c 2 \mathrm{DH}}$ is almost six times as much as the absolute value of $V_{x c 3 D}$ and $V_{x c 2 \mathrm{DL}}$. It is necessary to consider $V_{x c 2 \mathrm{DH}}$ in the research of interaction between incident charged particles and 2DQEG by using the QHD theory due to the high density in the quantum electron gas. This indicates that increasing density could enhance the exchange-correlation effect, as a function of the density. The exchange-correlation effect has been considered in quantum wells, GaAs/ GaAlAs heterostructures, and silicon inversion layers, with the local density approximation method $[49,50]$. 


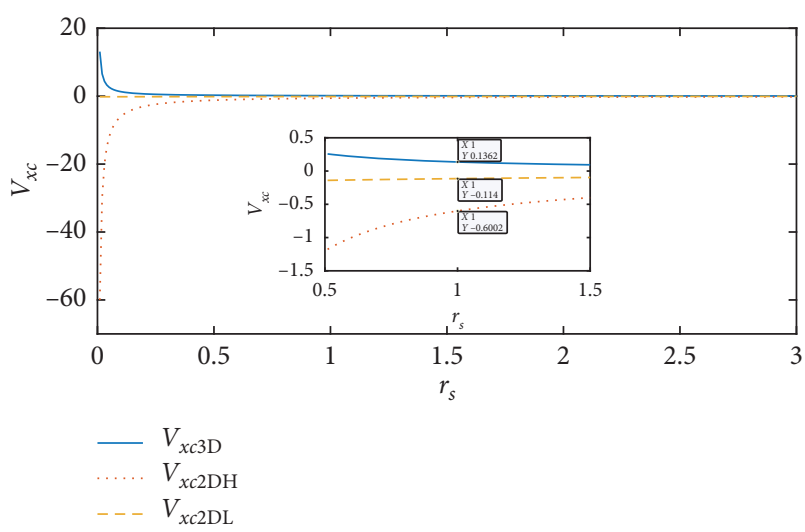

FIgURE 2: The change of electron exchange-correlation potential with Wigner-Seitz radius of 2DQEG.

2.4. Linearized QHD Model. Let $n_{e}(\mathbf{r}, t)=n_{0}+n_{e 1}(\mathbf{r}, t)$, $\mathbf{u}_{e}(\mathbf{r}, t)=\mathbf{u}_{e 1}(\mathbf{r}, t)$, and $\Phi(\mathbf{R}, t)=\Phi_{1}(\mathbf{R}, t)$, where $n_{e 1} \ll n_{0}$. $\mathbf{u}_{e 1}(\mathbf{r}, t), n_{e 1}(\mathbf{r}, t)$, and $\Phi_{1}(\mathbf{R}, t)$ are the first-order perturbed quantities of velocity, density, and potential, respectively. We linearize the above equation to obtain the perturbation electron density $n_{e 1}$ and the induced potential $\Phi_{\text {ind }}$ [5]. Here are the linearized continuity equation

$$
\frac{\partial n_{e 1}}{\partial t}+n_{0} \nabla_{\|} \cdot \mathbf{u}_{e 1}=0
$$

the momentum-balance equation

$$
\begin{aligned}
m_{e} \frac{\partial \mathbf{u}_{e 1}}{\partial t}= & \left.e \nabla_{\|} \Phi_{1}\right|_{z=0} \frac{\pi \hbar^{2}}{m_{e}} \nabla_{\|} n_{e 1}+\frac{\hbar^{2}}{4 m_{e} n_{0}} \nabla_{\|}\left(\nabla_{\|}^{2} n_{e 1}\right) \\
& -\gamma m_{e} \mathbf{u}_{e 1}-e E-V_{x c 2 \mathrm{DH}} \frac{\nabla n_{e 1}}{n_{0}}
\end{aligned}
$$

and Poisson's equation

$$
\nabla^{2} \Phi_{1}=4 \pi e\left[n_{e 1} \delta(z)-z_{1} \delta(\mathbf{r}-\mathbf{v} t) \delta\left(z-z_{0}\right)\right]
$$

where $\Phi_{1}=\Phi_{\text {ext }}+\Phi_{\text {ind }}$. $\Phi_{\text {ext }}$ is the external potential of the incident particle. $\Phi_{\text {ind }}$ is the induced potential by the perturbation electron density. Take the Fourier transform of the above linearized equations in space-time, and obtain the perturbation electron density $n_{e 1}$ and the induced potential $\Phi_{\text {ind }}$ :

$$
\begin{aligned}
n_{e 1}(\mathbf{r}, t)= & n_{0} \frac{z_{1} e^{2}}{2 \pi m_{e}} \int_{-\infty}^{+\infty}-\frac{k}{D(k, \omega)} e^{-k z_{0}} e^{i \mathbf{k} \cdot(\mathbf{r}-\mathbf{v} t)} \mathrm{d} \mathbf{k} \\
& -\frac{e k_{0}^{2} \Phi_{0} n_{0}}{m_{e}\left|D_{0}\left(k_{0}, \omega_{0}\right)\right|^{2}}\left\{\operatorname{Re}\left(D_{0}\left(k_{0}, \omega_{0}\right)\right) \cos \left(k_{0} x-\omega_{0} t\right)\right. \\
& \left.+\operatorname{Im}\left(D_{0}\left(k_{0}, \omega_{0}\right)\right) \sin \left(k_{0} x-\omega_{0} t\right)\right\}, \\
\Phi_{\text {ind }}(\mathbf{R}, t)= & n_{0} \frac{z_{1} e^{3}}{m_{e}} \int_{-\infty}^{+\infty} \frac{1}{D(k, \omega)} e^{-k z_{0}-k z} e^{i \mathbf{k} \cdot(\mathbf{r}-\mathbf{v} t)} \mathrm{d} \mathbf{k} \\
& +\frac{2 \pi e^{2} n_{0} k_{0} \Phi_{0}}{m_{e}\left|D_{0}\left(k_{0}, \omega_{0}\right)\right|^{2}}\left\{\operatorname{Re}\left(D_{0}\left(k_{0}, \omega_{0}\right)\right)\right. \\
& \cdot \cos \left(k_{0} x-\omega_{0} t\right)+\operatorname{Im}\left(D_{0}\left(k_{0}, \omega_{0}\right)\right) \\
& \left.\cdot \sin \left(k_{0} x-\omega_{0} t\right)\right\},
\end{aligned}
$$

where

$$
\begin{aligned}
D(k, \omega)= & \omega \widetilde{\omega}-\omega_{p}^{2} a_{B} k-\frac{V_{x c 2 \mathrm{DH}}}{m_{e}} k^{2} \\
& -\left(k v_{F}\right)^{2} \frac{\left(1+k^{2} /\left(2 k_{F}^{2}\right)\right)}{2}, \\
D_{0}\left(k_{0}, \omega_{0}\right)= & \omega_{0} \widetilde{\omega}_{0}-\omega_{p}^{2} a_{B} k_{0}-\frac{V_{x c 2 \mathrm{DH}}}{m_{e}} k_{0}^{2} \\
& -\left(k_{0} v_{F}\right)^{2} \frac{\left(1+k_{0}^{2} /\left(2 k_{F}^{2}\right)\right)}{2},
\end{aligned}
$$

with $\widetilde{\omega}=\omega+i \gamma$. Bohr radius $a_{B}=\hbar^{2} / m_{e} e^{2}$, Fermi velocity $v_{F}=\hbar k_{F} / m_{e}$, Fermi wave-number $k_{F}=\sqrt{2 \pi n_{0}}$, and electron plasma frequency $\omega_{p}=\left(2 \pi n_{0} e^{2} / m_{e} a_{B}\right)^{1 / 2} \cdot \mathbf{k}=\left\{k_{x}, k_{y}\right\}$ is a two-dimensional wave vector. Consider that $\omega=\mathbf{k} \cdot \mathbf{v}$ and the projectile velocity $\mathbf{v}$ and the $\mathbf{x}$ axis are going in the same direction, hence $\omega=k_{x} v$.

For convenience, we introduce the dimensionless variables: $\quad \omega / \omega_{p} \longrightarrow \omega, \quad \omega_{0} / \omega_{p} \longrightarrow \omega_{0}, \quad k_{y} / k_{F} \longrightarrow k_{y}$, $k / k_{F} \longrightarrow k, \quad k_{0} / k_{F} \longrightarrow k_{0}, \quad v / v_{B} \longrightarrow v, \quad c / v_{B} \longrightarrow c$, $(x-v t) / \lambda_{f} \longrightarrow x, y / \lambda_{f} \longrightarrow y, z / \lambda_{f} \longrightarrow z, z_{0} / \lambda_{f} \longrightarrow z_{0}$, $\gamma / \omega_{p} \longrightarrow \gamma$, and $\Phi_{0} /\left(e / \lambda_{f}\right) \longrightarrow \Phi_{0}$. Thus, equation (11) can be reduced to

$$
\begin{aligned}
\frac{n_{e 1}(\mathbf{r}, t)}{n_{0}}= & A_{n} \int_{-\infty}^{+\infty} \mathrm{d} k_{y} \int_{-\infty}^{+\infty} \mathrm{d} \omega B_{n} \cos \left(y k_{y}\right) \times\{\operatorname{Re}(\widetilde{D}) \cos (x \omega / v)+\operatorname{Im}(\widetilde{D}) \sin (x \omega / v)\} \\
& +C_{n}\left[\operatorname{Re}\left(\widetilde{D_{0}}\left(k_{0}, \omega_{0}\right)\right) \cos \left(x k_{0}+\theta\right)+\operatorname{Im}\left(\widetilde{D_{0}}\left(k_{0}, \omega_{0}\right)\right) \sin \left(x k_{0}+\theta\right)\right],
\end{aligned}
$$


where $A_{n}=\left(-Z_{1}\right) /\left(2 \pi r_{s} v\right), B_{n}=\left(k e^{-k z_{0}}\right) /\left(|\widetilde{D}|^{2}\right), C_{n}=\left(\Phi_{0}\right.$ $\left.k_{0}^{2}\right) /\left(\left|\widetilde{D_{0}}\right|^{2} r_{s}\right)$

$$
\begin{gathered}
\widetilde{D}=\omega^{2}-\frac{k}{r_{s}}-\left(\frac{k^{2}}{\left(2 r_{s}^{2}\right)}\right)\left(\frac{1+k^{2}}{2}\right)-V_{x c 2 \mathrm{DH}} k^{2}, \\
\widetilde{D_{0}}=\omega_{0}^{2}-\frac{k_{0}}{r_{s}}-\left(k_{0}^{2} / 24 r_{s}^{2}\right)\left(\frac{1+k_{0}^{2}}{2}\right)-V_{x c 2 \mathrm{DH}} k_{0}^{2},
\end{gathered}
$$

Here, $t$ denotes time variable, $\omega_{0}$ is constant when the wavelength $\lambda$ is fixed, and $\theta=-\omega_{0} t$ is used to modulate the phase, and it highlights the sine function factor oscillation of pump waves. Note that the introduced $V_{x c 2 \mathrm{DH}}$ affects the electron density distribution by modifying the value of $\widetilde{D}$, determined through the Wigner-Seitz radius $r_{s}$ thus by the equilibrium density $n_{0}$. The second term of the reduced perturbation electron density is induced by the electric field of the pump wave in equation (14). Because the pump wave satisfies the relation $k_{0}=1 / \lambda$, the second term of the reduced perturbation electron density is modulated by the wavelength $\lambda$, the amplitude $\Phi_{0}$, and the time phase $\theta$. Furthermore, the stopping power $S(v)$ determined by wake force can be given by the value of the gradient of the induced potential $\Phi_{\text {ind }}[32]$ :

$$
\begin{aligned}
S(v)= & \left.e Z_{1} \frac{\partial \Phi_{\text {ind }}}{\partial x}\right|_{z=z_{0}} ^{\mathbf{r}=\mathbf{v} t} \\
= & n_{0} \frac{z_{1}^{2} e^{4}}{m_{e}} \int_{-\infty}^{+\infty} \frac{i k_{x}}{D(k, \omega)} e^{-k z_{0}-k z} e^{i \mathbf{k} \cdot(\mathbf{r}-\mathbf{v} t)} \mathrm{d} \mathbf{k} \\
& +\frac{2 \pi z_{1} e^{3} n_{0} k_{0}^{2} \Phi_{0} e^{-k_{0} z}}{m_{e}\left|D_{0}\left(k_{0}, \omega_{0}\right)\right|^{2}} \\
& \left\{\operatorname{Im}\left(D_{0}\left(k_{0}, \omega_{0}\right)\right) \cos \left(k_{0} x-\omega_{0} t\right)\right. \\
& \left.-\operatorname{Re}\left(D_{0}\left(k_{0}, \omega_{0}\right)\right) \sin \left(k_{0} x-\omega_{0} t\right)\right\} .
\end{aligned}
$$

In the calculation above, the residue theorem is used. Using dimensionless variables, equation (16) can be reduced to

$$
\begin{aligned}
\frac{S(v)}{S_{0}}= & A_{s} \int_{-\infty}^{+\infty} \mathrm{d} k_{y} \int_{-\infty}^{+\infty} \mathrm{d} \omega B_{s} \operatorname{Im}\left[\widetilde{D}^{-1}\right] \\
& +C_{s}\left\{\operatorname{Im}\left(\widetilde{D_{0}}\left(k_{0}, \omega_{0}\right)\right) \cos \left[k_{0}(v-c)+\theta\right]\right. \\
& \left.-\operatorname{Re}\left(\widetilde{D_{0}}\left(k_{0}, \omega_{0}\right)\right) \sin \left[k_{0}(v-c)+\theta\right]\right\}
\end{aligned}
$$

where

$$
\begin{aligned}
& S_{0}=\frac{\left(Z_{1} e / a_{B}\right)^{2}}{2 \pi}, \\
& A_{s}=\frac{\left(v_{B}^{2}\right)}{\left(2 \pi r_{s}^{3} v^{2}\right)}, \\
& B_{s}=-\omega e^{-2 k z_{0}}, \\
& C_{s}=\frac{\Phi_{0} k_{0}^{2} e^{-k_{0} z_{0}}}{\left(r_{s}^{3}\left|\widetilde{D_{0}}\right|^{2}\right)} .
\end{aligned}
$$

The stopping power is related to the velocity $v$ of the incident particle. When the velocity variable $v$ is extracted from the sine and cosine functions, the time variable $t$ is also exposed. Similarly, to study the influence of the time variable $t$ on the sine and cosine functions, we introduce the time phase $\theta$, which means that the time variable $t$ is changing the sine and cosine functions with $-\gamma_{0} t$ in equation (16) while the time phase $\theta$ is changing the sine and cosine functions through $\theta$ in equation (17). Note that the second term of the stopping power is induced by the excited electric field of the pump wave in equation (16). The pump wave satisfies the relation $\omega_{0}=k_{0} c$ and $3 k_{0}=1 / \lambda$, and hence, the second term of the stopping power is modulated by the wavelength $\lambda$, the amplitude $\Phi_{0}$, and the time phase $\theta$.

In the next section, we will give the result of the effect of $V_{x c 2 \mathrm{DH}}$ on the stopping power of the incident particle. To investigate the effect of pump wave on the perturbation electron density of 2DQEG and the stopping power of the incident particle, we calculate the perturbation electron density and the stopping power under different wavelength $\lambda$, amplitude $\Phi_{0}$, and time phase $\theta$. In what follows, we take $r_{s}=1$, the collision frequency $\gamma=0.02 \omega_{P}$, incident particle as a proton $Z_{1}=1$, and the distance between the incident particle and 2DQEG $z_{0}=9 \lambda_{f}$, while the wavelength $\lambda$, the amplitude $\Phi_{0}$, and the time phase $\theta$ of the pump wave are varied.

\section{Result and Discussion}

3.1. Two-Dimensional Electron Exchange-Correlation Potential of High Density. In the past, Li et al. and Zhang et al. have done a lot of theoretical research studies on the stopping power of incident particles in the study of the charged particles moving above two-dimensional electron gases $[4,5,32,38,43]$. On the basis of the work of Li et al. and Zhang et al., we investigate the effect of exchange-correlation potential on the stopping power of incident particles. In this paper, the stopping power of incident particles calculated without considering the exchange-correlation potential is 
consistent with the research results of $\mathrm{Li}$ et al. and Zhang et al.

As it can be assumed, $V_{x c 2 \mathrm{DH}}$ modified the stopping power of the incident particle because $V_{x c 2 \mathrm{DH}}$ has been considered in the momentum-balance equation, thereby changing the value of $D$ and $D_{0}$ in equation (16) and modifying the stopping power of the incident particle. In order to verify the necessity of introducing $V_{x c 2 \mathrm{DH}}$ to modifying QHD, Figure 3 presents the stopping power of the incident particle versus particle velocity under different conditions.

Comparing the curve of stopping power without exchange-correlation potential (solid line), the curve of stopping power with $V_{x c 3 D}=0.1362$ (dashed line), the curve of stopping power with $V_{x c 2 \mathrm{DL}}=-0.114$ (dotted line), and the curve of stopping power with $V_{x c 2 \mathrm{DH}}=-0.6002$ (dashdotted line) are shown in Figure 3. Considering the peak of the stopping power of the incident particle near $V=3.5 V_{B}$ in Figure 3, the value of stopping power without exchangecorrelation potential exhibits an obvious difference with respect to stopping power featured by $V_{x c 3}, V_{x c 2 \mathrm{DL}}$, and $V_{x c 2 \mathrm{DH}}$. The value of stopping power without exchangecorrelation potential is $0.01517 S_{0}$. The values of stopping power with $V_{x c 3 D}, V_{x c 2 \mathrm{DL}}$, and $V_{x c 2 \mathrm{DH}}$, respectively, are $0.01506 S_{0}, 0.01525 S_{0}$, and $0.01559 S_{0}$, which, respectively, are changed by $0.725 \%, 0.527 \%$, and $2.77 \%$ relative to stopping power without $V_{x c}$ (these are dimensionless values). The results show that the two-dimensional exchange-correlation potential, especially $V_{x c 2 \mathrm{DH}}$, influences the stopping power of incident particles. $V_{x c 2 \mathrm{DH}}$ is adapted throughout the paper within subsequent results.

3.2. Amplitude of Pump Wave. In order to study the modulation of pump wave amplitude $\Phi_{0}$ on the perturbation electron density and the stopping power, Figures 4 and 5 show the spatial distribution of the perturbation electron density as a function of $x$ and the stopping power of the incident particle versus particle velocity under three different intensity conditions of the pump wave, where the wavelength is $\lambda=1.67 \times 10^{-8} \mathrm{~cm}$. The comparison of the perturbation electron density without the pump wave and with the pump wave for $\Phi_{0}=1 \times 10^{2} e / \lambda_{f}, \Phi_{0}=2 \times 10^{2} e / \lambda_{f}$, and $\Phi_{0}=3 \times 10^{2} e / \lambda_{f}$, respectively, shows that the oscillation amplitude of the perturbation electron density increases when considering the pump wave in most cases and the oscillation amplitude of the perturbation electron density becomes larger as the amplitude of the pump wave increases in Figure 4. This is because the value of the second term in equation (14) is proportional to the factor of $\Phi_{0}$. But the oscillation amplitude of the perturbation electron density displays specific features near $x=-29 \lambda_{f}$. This is due to the value of the incident particle external potential and induced potential by the perturbation electron density. The comparison of the stopping power without the pump wave and with the pump wave for $\Phi_{0}=1 \times 10^{2} e / \lambda_{f}, \Phi_{0}=2 \times 10^{2} e / \lambda_{f}$, and $\Phi_{0}=3 \times 10^{2} e / \lambda_{f}$, respectively, shows that the pump wave yields obvious modulation effects on the stopping power, and the intensity of this modulation is proportional

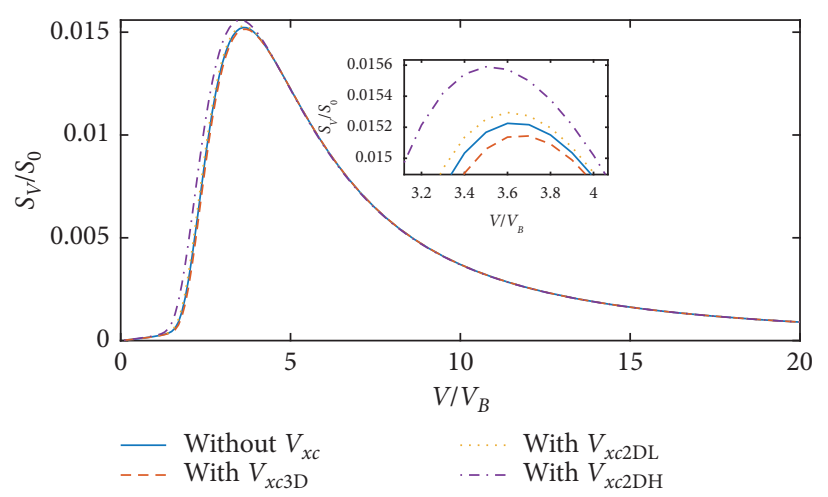

FIgURE 3: The stopping power of incident particles, respectively, with $V_{x c 3 D}=0.1362, V_{x c 2 \mathrm{DH}}=-0.6002$, and $V_{x c 2 \mathrm{DL}}=-0.114$. Here, $Z_{1}=1, r_{s}=1, \gamma=0.02$, and $Z_{0}=9$.

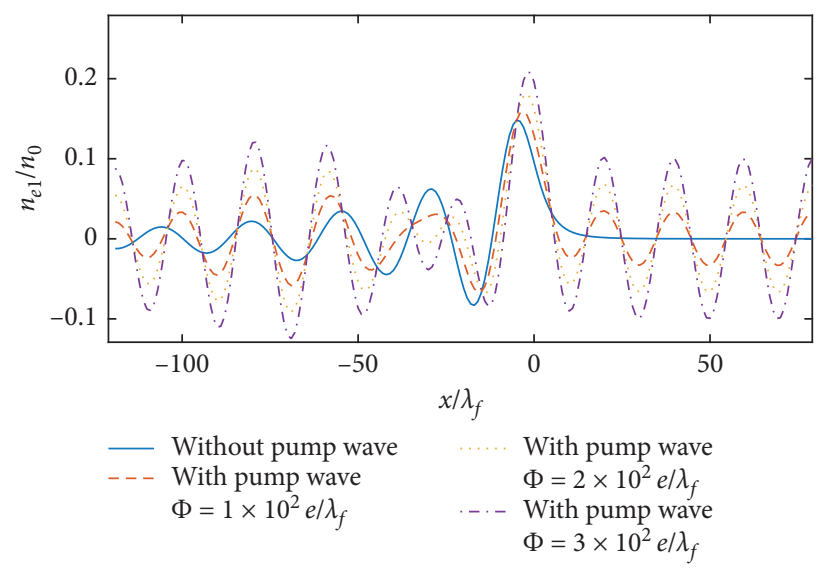

FIgURE 4: 2DQEG perturbation electron density. Here, $Z_{1}=1, r_{s}=$ $1, v=2, \gamma=0.02, Z_{0}=9, \lambda=1.67 \times 10^{-8} \mathrm{~cm}$, and $\theta=0$.

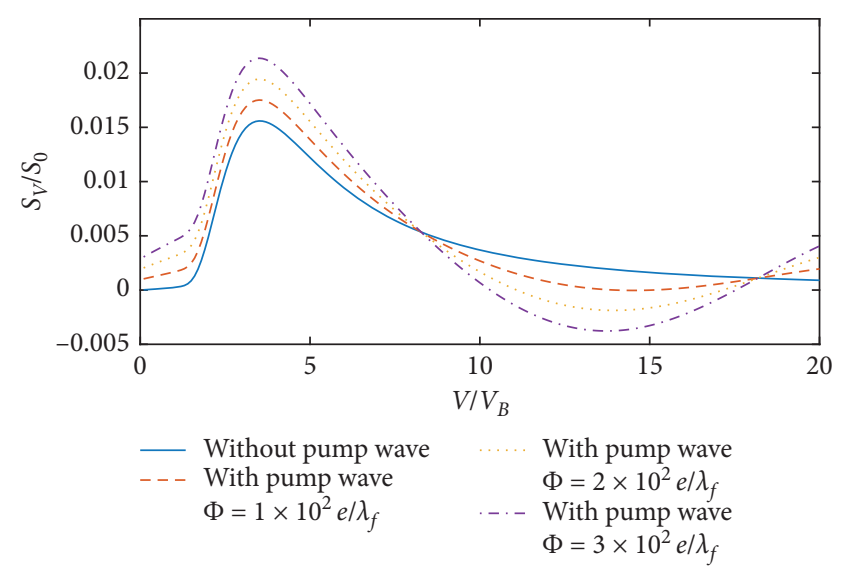

FIGURE 5: Stopping power of the incident charged particle. Here, $Z_{1}=1, r_{s}=1, \gamma=0.02, Z_{0}=9, \lambda=1.67 \times 10^{-8} \mathrm{~cm}$, and $\theta=0$.

to the amplitude of the pump wave in Figure 5. This is because the value of the second term in equation (17) is proportional to $\Phi_{0}$. In the low-velocity region $\left(v<8.3 v_{B}\right)$, the stopping power that peaks at $v=3.5 v_{B}$ with the pump wave for $\Phi_{0}=1 \times 10^{2} e / \lambda_{f}, \Phi_{0}=2 \times 10^{2} e / \lambda_{f}$, and $\Phi_{0}=3 \times 10^{2} e / \lambda_{f}$, 
respectively, increases by $12.4 \%, 24.7 \%$, and $37.1 \%$ relative to the case without the pump wave. Moreover, in the high-velocity region $\left(v>8.3 v_{B}\right)$, it is easy to see that the pump wave dominates the stopping power as the particle velocity increases compared to the stopping power quickly vanishing without the pump wave. The value of stopping power at $v=13.7 v_{B}$ with pump wave for $\Phi_{0}=3 \times 10^{2} \mathrm{e} / \lambda_{f}$ decreases to $-0.003772 S_{0}$. It is surprising that a negative stopping power [34] is found due to the existence of the pump wave, different from our observation without the pump wave. The appearance of negative stopping power means that the particle drains energy from the 2DQEG.

Generally, when the pump wave does not exist, due to the disturbance of the electron density in 2DQEG caused by charged particles, negative charges will accumulate behind the charged particles in 2DQEG to excite the wake field, thereby reducing the speed of the charged particles. When the pump wave exists, in addition to the density disturbance of 2DQEG caused by charged particles, we also need to consider the density disturbance caused by the electric field component of the pump wave. Since the electric field of the pump wave changes periodically with time, the density disturbance caused by the electric field component of the pump wave will also change periodically. The deceleration and acceleration effects of the electric field excited by the perturbation density caused by the pump wave on the charged particles will change periodically with time. When the electric field excited by the perturbation density caused by the pump wave in 2DQEG has a deceleration effect on the charged particles, the stopping power of the charged particles will increase. When the electric field excited by the perturbation density caused by the pump wave accelerates the charged particles, the stopping power of the charged particles will decrease. When the acceleration of the electric field excited by the perturbation density caused by the pump wave has a stronger effect on the charged particles than the deceleration of wake field excited by the charged particles, the velocity of the charged particles will increase and negative stopping power appears. In other words, the polarity of wake field can be tuned in the presence of the pump wave, yielding the negative stopping power.

In the case of $\lambda=1.56 \times 10^{-7} \mathrm{~cm}$, the comparison of the perturbation electron density and stopping power without the pump wave and with the pump wave shows that the modulation effect on the oscillation amplitude of the perturbation electron density and stopping power is proportional to the amplitude of the pump wave in Figures 6 and 7. As the value of the second term in equations (14) and (17) increases with increase in $\Phi_{0}$, it is easy to see that the oscillation amplitude of the perturbation electron density at $x=-5 \lambda_{f}$ with the pump wave for $\Phi_{0}=1 \times 10^{2} e / \lambda_{f}, \quad \Phi_{0}=2 \times 10^{2} e / \lambda_{f}, \quad$ and $\Phi_{0}=3 \times 10^{2} e / \lambda_{f}$, respectively, increases by $22.2 \%, 44.5 \%$, and $66.8 \%$ in Figure 6 . At $x>10 \lambda_{f}$ or $x<-46 \lambda_{f}$, the pump wave with wavelength $\lambda=1.56 \times 10^{-7} \mathrm{~cm}$ dominates the oscillation amplitude of the perturbation electron density compared to the perturbation electron density quickly vanishing without the pump wave. The stopping power at $v=$ $3.5 v_{B}$ with the pump wave for $\Phi_{0}=1 \times 10^{2} e / \lambda_{f}$, $\Phi_{0}=2 \times 10^{2} e / \lambda_{f}$, and $\Phi_{0}=3 \times 10^{2} e / \lambda_{f}$, respectively, increases to $0.03991 S_{0}, 0.06422 S_{0}$, and $0.08854 S_{0}$ in Figure 7.

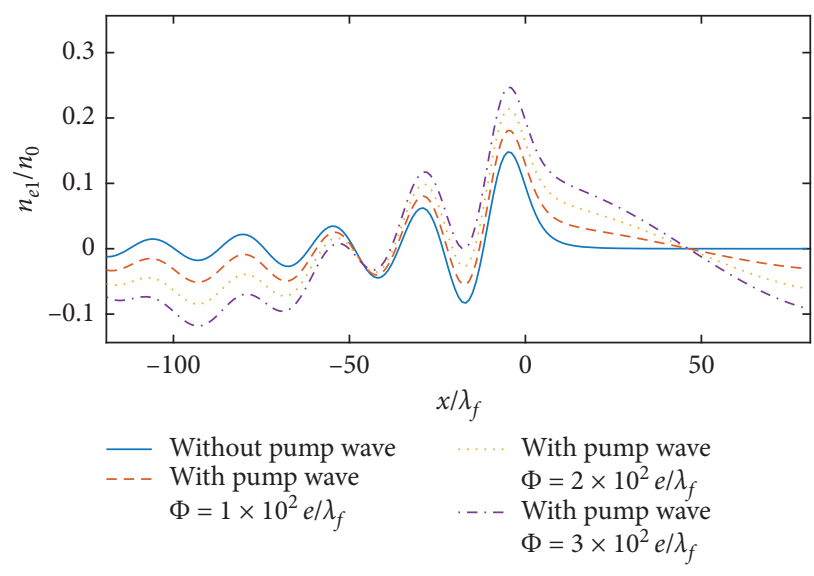

FIgURE 6: 2DQEG perturbation electron density. Here, $Z_{1}=1, r_{s}=$ $1, v=2, \gamma=0.02, Z_{0}=9, \lambda=1.56 \times 10^{-7} \mathrm{~cm}$, and $\theta=0$.

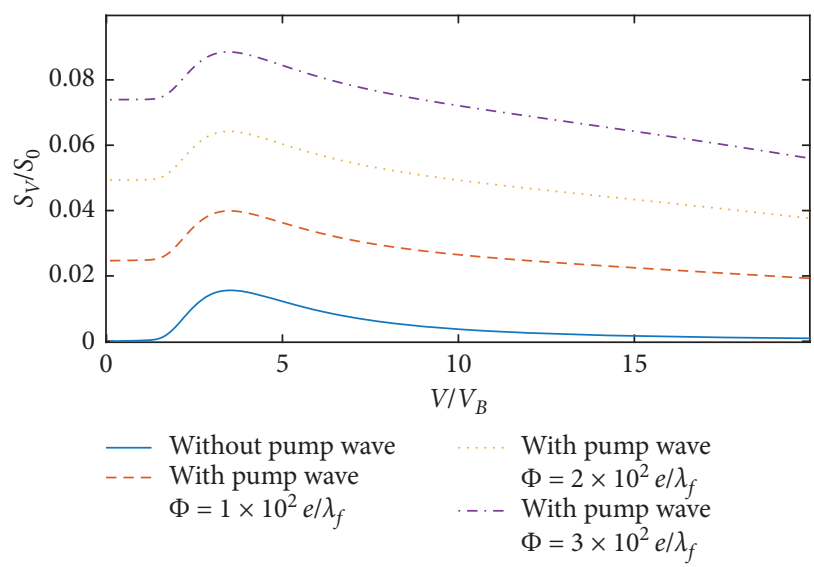

FIGURE 7: Stopping power of the incident charged particle. Here, $Z_{1}=1, r_{s}=1, \gamma=0.02, Z_{0}=9, \lambda=1.56 \times 10^{-7} \mathrm{~cm}$, and $\theta=0$.

In the case of $\lambda=1.76 \times 10^{-6} \mathrm{~cm}$, the comparison of the perturbation electron density and stopping power without the pump wave and with the pump wave shows that the pump wave has an obvious modulation effect on the oscillation amplitude of the perturbation electron density and stopping power in Figures 8 and 9, and the modulation effect of pump wave strengthens as the amplitude of pump wave increases, which again arises from equations (14) and (17) second term proportional to $\Phi_{0}$. The modulation effect of the pump wave only changes the amplitude, not the shape of the perturbation electron density and stopping power. It is easy to see that the oscillation amplitude of the perturbation electron density at $x=-5 \lambda_{f}$ with the pump wave for $\Phi_{0}=1 \times 10^{2} e / \lambda_{f}, \Phi_{0}=2 \times 10^{2} e / \lambda_{f}$, and $\Phi_{0}=3 \times 10^{2} e / \lambda_{f}$, respectively, increases by $22.9 \%, 45.8 \%$, and $68.7 \%$ in Figure 8 . The oscillation amplitude of the perturbation electron density increases with the pump wave amplitude. The amplitude of the stopping power at $v=3.5 v_{B}$ with pump wave for $\Phi_{0}=1 \times 10^{2} e / \lambda_{f}, \Phi_{0}=2 \times 10^{2} e / \lambda_{f}$, and $\Phi_{0}=3 \times 10^{2} e$ $/ \lambda_{f}$, respectively, decreases to $0.004182 S_{0},-0.0007226 S_{0}$, and $-0.01862 S_{0}$ in Figure 9. The oscillation amplitude of the 


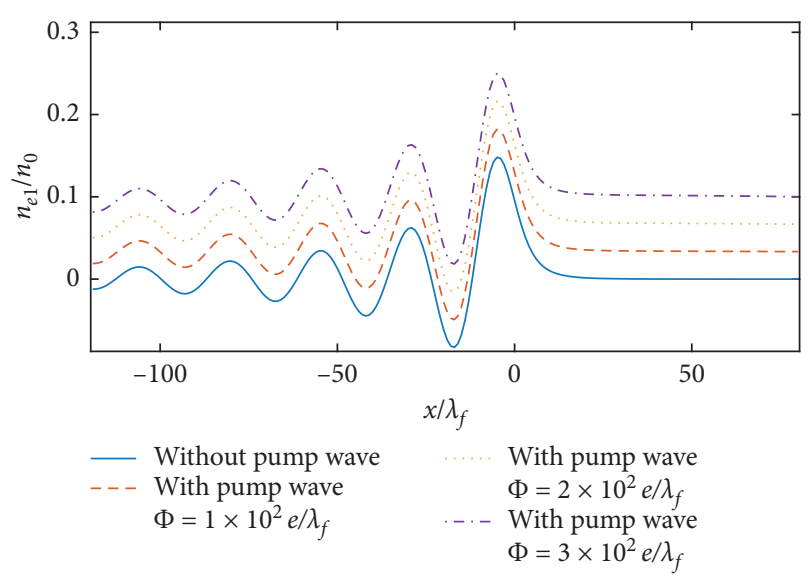

FIgURE 8: 2DQEG perturbation electron density. Here, $Z_{1}=1, r_{s}=$ $1, v=2, \gamma=0.02, Z_{0}=9, \lambda=1.76 \times 10^{-6} \mathrm{~cm}$, and $\theta=0$.

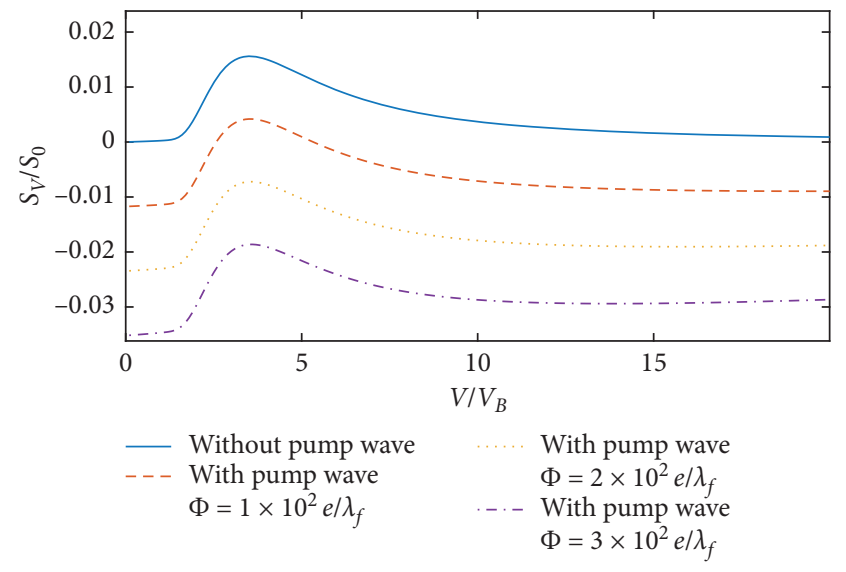

FIGURE 9: Stopping power of the incident charged particle. Here, $Z_{1}=1, r_{s}=1, \gamma=0.02, Z_{0}=9, \lambda=1.76 \times 10^{-6} \mathrm{~cm}$, and $\theta=0$.

stopping power rises as the amplitude of the pump wave increases. Interestingly, the negative stopping power is also found in Figure 9.

Figures $10-15$ show the perturbation electron density and stopping power with the pump wave for $\lambda=1.76 \times 10^{-4}$ $\mathrm{cm}, \lambda=1.76 \times 10^{-2} \mathrm{~cm}$, and $\lambda=1.76 \times 10^{-1} \mathrm{~cm}$. The results of Figures 12 and 14 are similar to that of Figure 10. And the results of Figures 13 and 15 are similar to that of Figure 11 because the variations of the second term in equations (14) and (17) remain small in the domain of $-120 \lambda_{f}<x>80 \lambda_{f}$ and $0<v>20 v_{B}$. The oscillation amplitude of the perturbation electron density increases as the amplitude of pump wave rises in Figures 10, 12, and 14. The effect of the pump wave on the stopping power strengthens as the amplitude of pump wave increases in Figures 11, 13, and 15.

Therefore, the amplitude of the pump wave has a significant effect on the oscillation amplitude of the perturbation electron density and stopping power, while the oscillation amplitude of the perturbation electron density and stopping power rises with the pump wave amplitude.

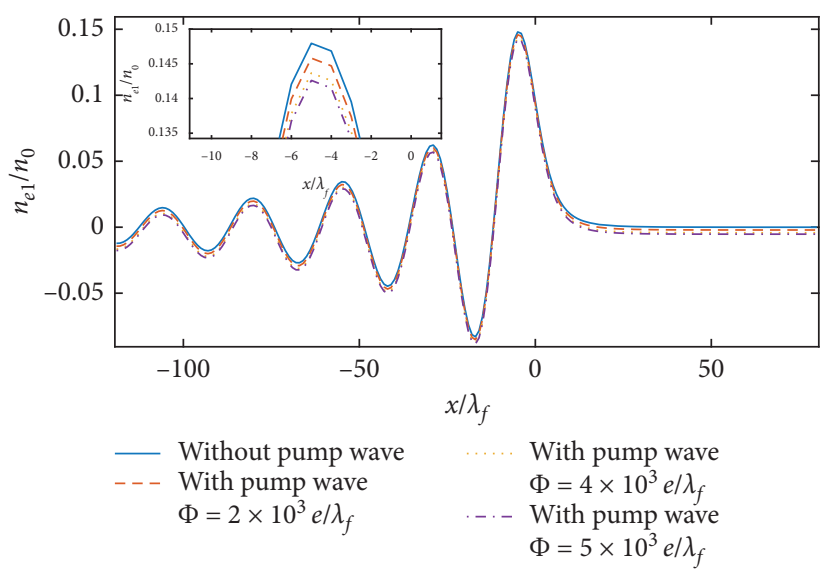

FIGURE 10: 2DQEG perturbation electron density. Here, $Z_{1}=1, r_{s}=1, v=2, \gamma=0.02, Z_{0}=9, \lambda=1.76 \times 10^{-4} \mathrm{~cm}$, and $\theta=0$.

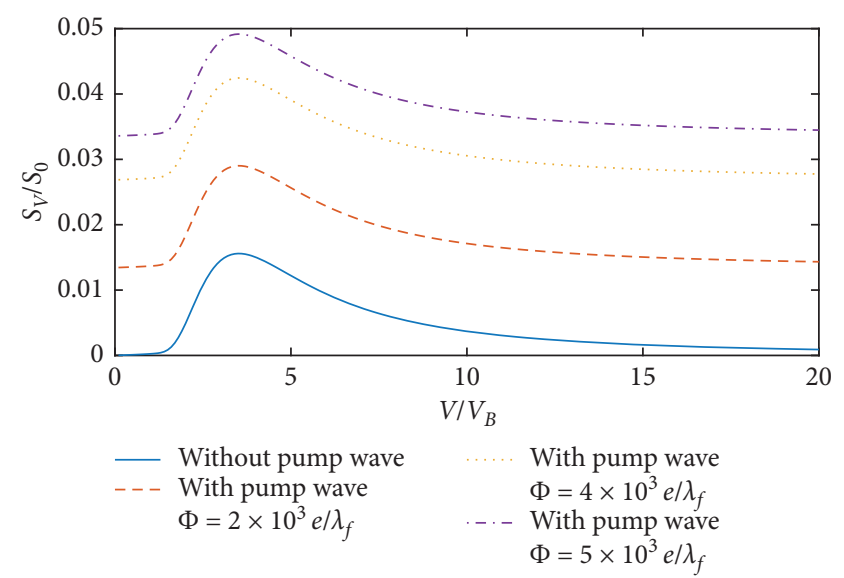

FIGURE 11: Stopping power of the incident charged particle. Here, $Z_{1}=1, r_{s}=1, \gamma=0.02, Z_{0}=9, \lambda=1.76 \times 10^{-4} \mathrm{~cm}$, and $\theta=0$.

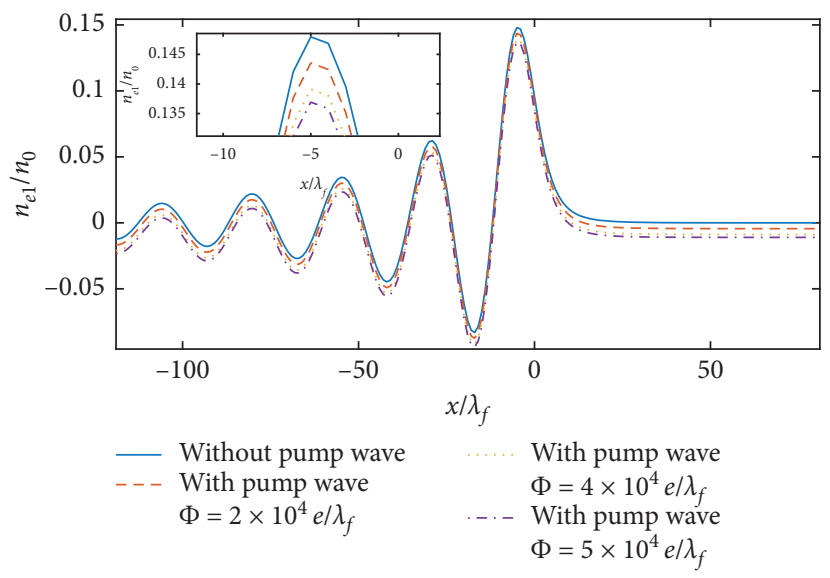

FIgURE 12: 2DQEG perturbation electron density. Here, $Z_{1}=1, r_{s}=1, v=2, \gamma=0.02, Z_{0}=9, \lambda=1.76 \times 10^{-2} \mathrm{~cm}$, and $\theta=0$.

The amplitudes $\Phi_{0} \leq 5 \times 10^{5} e / \lambda_{f}$ are realistic values in current experimental technologies and consistent with those used in [51]. 

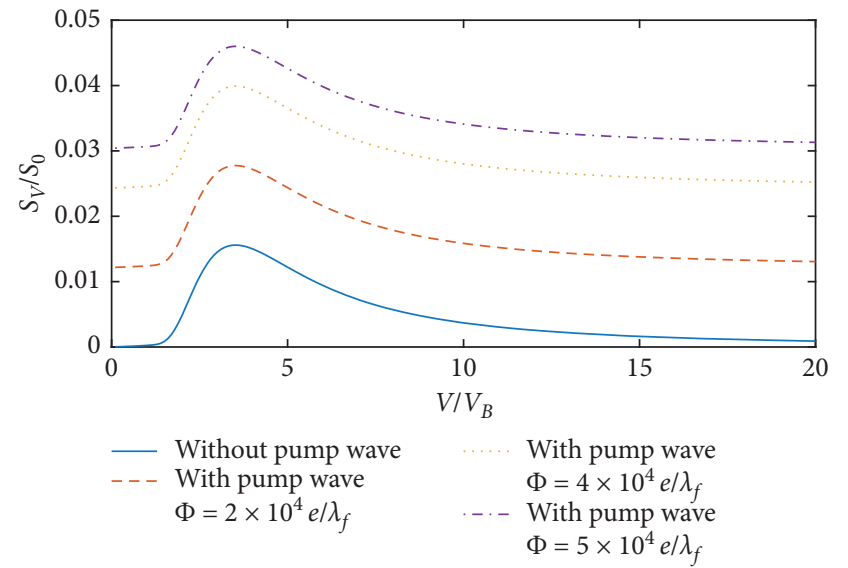

Figure 13: Stopping power of the incident charged particle. Here, $Z_{1}=1, r_{s}=1, \gamma=0.02, Z_{0}=9, \lambda=1.76 \times 10^{-2} \mathrm{~cm}$, and $\theta=0$.

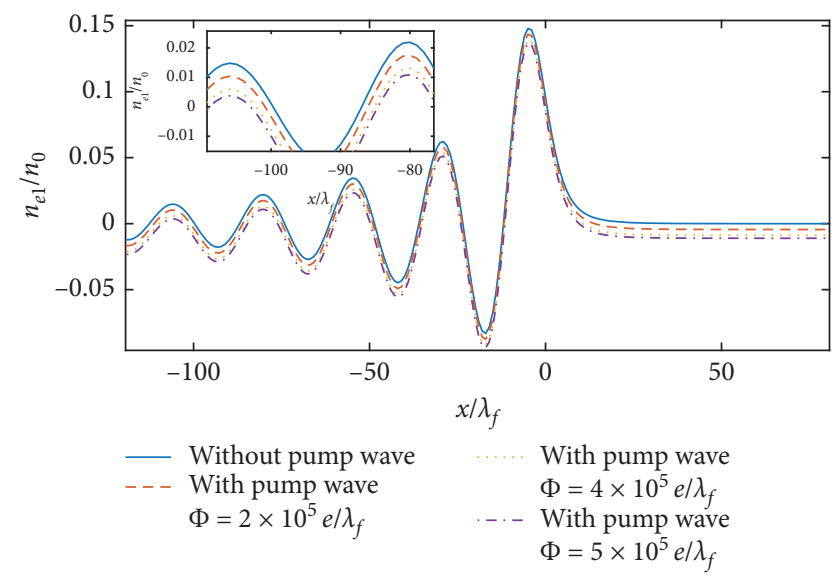

FIgURE 14: 2DQEG perturbation electron density. Here, $Z_{1}=1, r_{s}=1, v=2, \gamma=0.02, Z_{0}=9, \lambda=1.76 \times 10^{-1} \mathrm{~cm}, \quad$ and $\theta=0$.

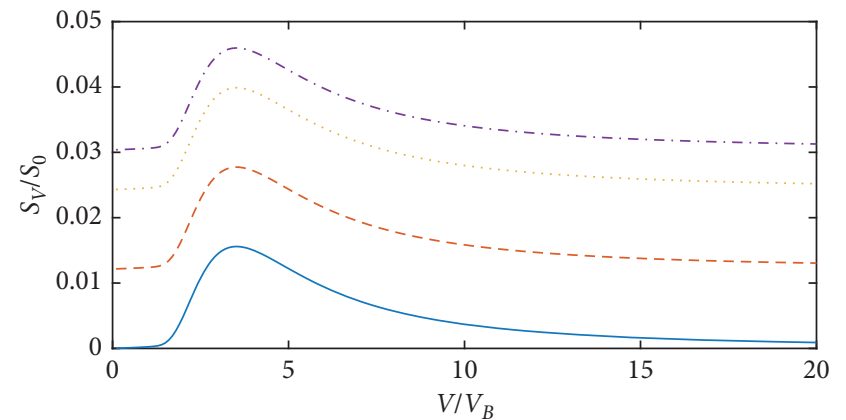

$$
\begin{aligned}
& \begin{array}{ll}
\text { - Without pump wave } & \text { With pump wave } \\
\text { - - With pump wave } & \Phi=4 \times 10^{5} e / \lambda_{f}
\end{array} \\
& \Phi=2 \times 10^{5} \mathrm{e} / \lambda_{f} \quad \text {... With pump wave } \\
& \Phi=5 \times 10^{5} \mathrm{e} / \lambda_{f}
\end{aligned}
$$

FIgURE 15: Stopping power of the incident charged particle. Here, $Z_{1}=1, r_{s}=1, \gamma=0.02, Z_{0}=9, \lambda=1.76 \times 10^{-1} \mathrm{~cm}$, and $\theta=0$.

3.3. Wavelength of the Pump Wave. The wavelength of the pump wave is an essential parameter for the modulation of the pump wave on the perturbation electron density and the stopping power. Comparing Figures 14 and 15, it is found that the influence of wavelength of the pump wave on the perturbation electron density and the stopping power is mainly reflected in the modulation of the shape of the perturbation electron density curve and the stopping power curve.

Let us consider the perturbation electron density curve and the stopping power curve for a short wavelength of the pump wave of $\lambda=1.67 \times 10^{-8} \mathrm{~cm}$. In the space range from $-120 \lambda_{f}$ to $80 \lambda_{f}$, the perturbation electron density shows a violent periodic oscillation, contrasted to the no pump wave case, as shown in Figure 4. The stopping power also has been modulated by the pump wave in the velocity range from $0 v_{B}$ to $20 v_{B}$ in Figure 5. The reason is that the second term contains the factor $k_{0}$ in equations (14) and (17), which is inversely proportional to the wavelength $\lambda$. So the perturbation electron density and the stopping power are strongly modulated by the pump wave.

Observe the perturbation electron density curve and the stopping power curve with a wavelength of $\lambda=1.56 \times 10^{-7}$ $\mathrm{cm}$. The number of oscillating periods of the perturbation electron density decreases over a range of $-120 \lambda_{f}$ to $80 \lambda_{f}$ in Figure 6. The oscillation period of the stopping power is smaller than one period in the velocity range from $0 v_{B}$ to $20 v_{B}$ in Figure 7. This is because the $k_{0}$ value decreases in equations (14) and (17) as the wavelength $\lambda$ is increasing. Therefore, the effect of the pump wave on the perturbation electron density and stopping power modulation is reduced compared to the wavelength $\lambda=1.67 \times 10^{-8} \mathrm{~cm}$.

With the wavelength of the pump wave $\lambda=1.76 \times 10^{-6}$ $\mathrm{cm}$, the perturbation electron density oscillates less than one period in the space range of $-120 \lambda_{f}$ to $80 \lambda_{f}$ in Figure 8 . The stopping power oscillation period is also shorter than one period in the velocity range from $0 v_{B}$ to $20 v_{B}$ in Figure 9. As the wavelength $\lambda$ becomes longer, the value of $k_{0}$ becomes smaller. The pump wave modulation to perturbation electron density and stopping power is further reduced in the region of $-120 \lambda_{f}<x<80 \lambda_{f}$ and $0<v<20 v_{B}$.

When the amplitude of the pump wave is $\Phi_{0}=3 \times 10^{2} e / \lambda_{f}$, the maximum value of perturbation electron density curves with wavelength $\lambda=1.67 \times 10^{-8} \mathrm{~cm}$, $\lambda=1.56 \times 10^{-7} \mathrm{~cm}$, and $\lambda=1.76 \times 10^{-6} \mathrm{~cm}$, respectively, is $0.2082 n_{0}, 0.2469 n_{0}$, and $0.2497 n_{0}$ in Figures 4,6 , and 8 . Those results show that the maximum value of the perturbation electron density increases in the region of $-120 \lambda_{f}<x<80 \lambda_{f}$ with the wavelength which means that the wavelength of the pump wave not only modifies the shape of the curve but also the amplitude of the perturbation electron density. Comparing the stopping power curves when the amplitude of pump wave is $\Phi_{0}=3 \times 10^{2} e / \lambda_{f}$ in Figures 5, 7, and 9, the maximum value of stopping power with wavelength $\lambda=1.67 \times 10^{-8} \mathrm{~cm}, \lambda=1.56 \times 10^{-7} \mathrm{~cm}$, and $\lambda=1.76 \times 10^{-6} \mathrm{~cm}$, respectively, is $0.02137 S_{0}$, $0.08854 S_{0}$, and $-0.01863 S_{0}$. The value of stopping power dramatically changes because $k_{0}$ varied when the wavelength is increasing.

As the wavelength of the pump wave increases, the pump wave modulates the shape of the perturbation electron density curve more and more weakly in the space range of 


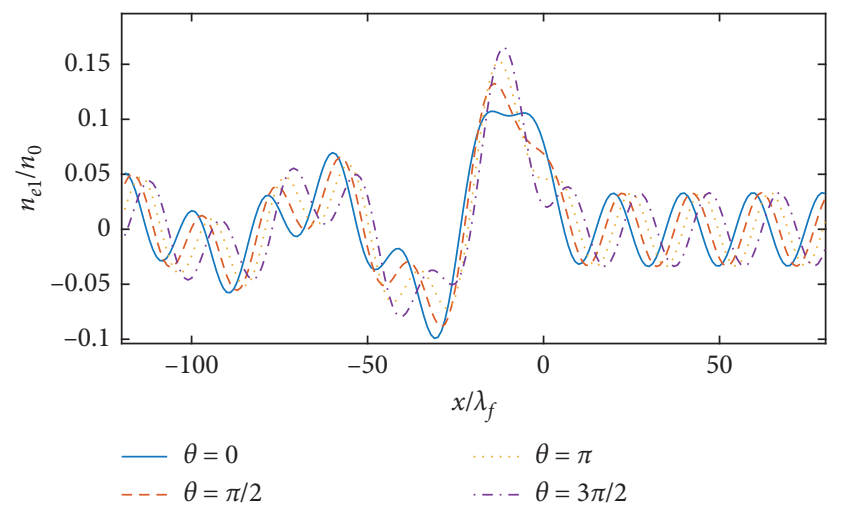

FIgURE 16: 2DQEG perturbation electron density. Here, $Z_{1}=1$, $r_{s}=1, \quad v=2, \quad \gamma=0.02, \quad Z_{0}=9, \quad \lambda=1.67 \times 10^{-8} \mathrm{~cm}, \quad$ and $\Phi_{0}=1 \times 10^{2} e / \lambda_{f}$.

$-120 \lambda_{f}$ to $80 \lambda_{f}$ in Figures 10, 12, and 14. Similarly, as the wavelength of the pump wave increases, the shape of the stopping power curve is modulated more weakly by the pump waves in the $0 v_{B}$ to $20 v_{B}$ speed range in Figures 11, 13, and 15. The modulation of the wavelength of the pump wave to the shape of the perturbation electron density curve and the stopping power curve decreases as the wavelength increases, arising from the tiny second term change in equations (14) and (17).

3.4. Phase of Pump Wave. Finally, in order to study the effect of time phase $\theta$ on the perturbation electron density and stopping power, Figures 16-19 exhibit the perturbation electron density and stopping power with wavelength $\lambda=$ $1.67 \times 10^{-8} \mathrm{~cm}$ and $\lambda=1.76 \times 10^{-4} \mathrm{~cm}$ for $\theta=0$ (solid line), $\theta=\pi / 2$ (dashed line), $\theta=\pi$ (dotted line), and $\theta=3 \pi / 2$ (dash-dotted line). Here, $\Phi_{0}=1 \times 10^{2} e / \lambda_{f}$. It is easy to see that the amplitude of perturbation electron density and stopping power oscillates periodically with different time phase $\theta$ in Figures 16-19. This is because the second term includes the parameter time phase $\theta$ in equations (14) and (17). Therefore, the time phase $\theta$ obviously impacts the perturbation electron density and stopping power.

It is worth pointing out that the negative stopping power in Figures 5, 9, and 19 we found is instantaneous, which is difficult to verify experimentally, and it is also difficult to apply. So we consider averaging the stopping power with respect to the time phase $\theta$. After averaging the stopping power with respect to the time phase $\theta$ from 0 to $2 \pi$, we found that the pump wave with $\Phi_{0}=1 \times 10^{2} e / \lambda_{f}$ decreases the value of stopping power in Figure 20. This is because the value of averaged second term is negative in equation (17) with respect to the time phase $\theta$. But amplitude reduction shows up as a relatively small one in connection to the too low pump wave intensity (Figure 20). This is because the intensity of pump wave is too low. Linearized QHD is valid only for low intensity of pump wave. The comparison of the stopping power without the pump wave and the average stopping power with pump wave for $\Phi_{0}=2 \times 10^{10} e / \lambda_{f}$ and $\lambda=1.76 \times 10^{-4} \mathrm{~cm}$ demonstrates that the average stopping power decreases in the region of $0<v<20 V_{B}$ in Figure 20.

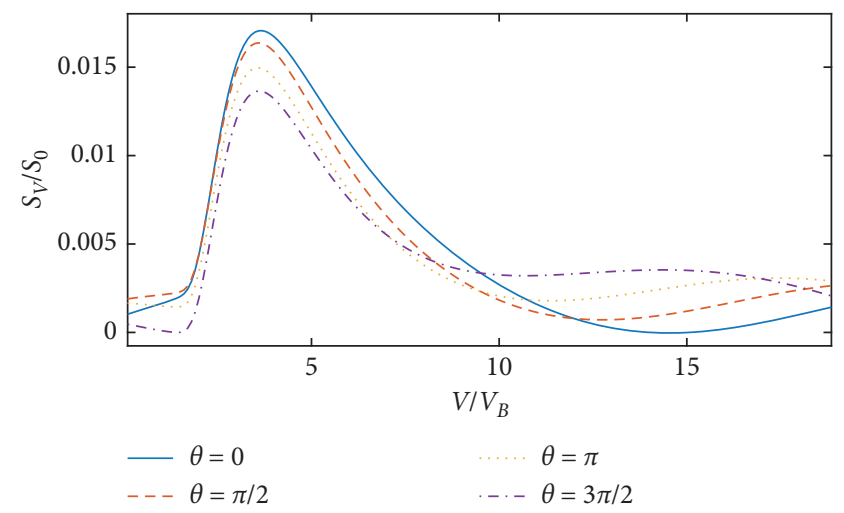

FIGURE 17: Stopping power of the incident charged particle. Here, $Z_{1}=1, \quad r_{s}=1, \quad \gamma=0.02, \quad Z_{0}=9, \quad \lambda=1.67 \times 10^{-8} \mathrm{~cm}, \quad$ and $\Phi_{0}=1 \times 10^{2} e / \lambda_{f}$.

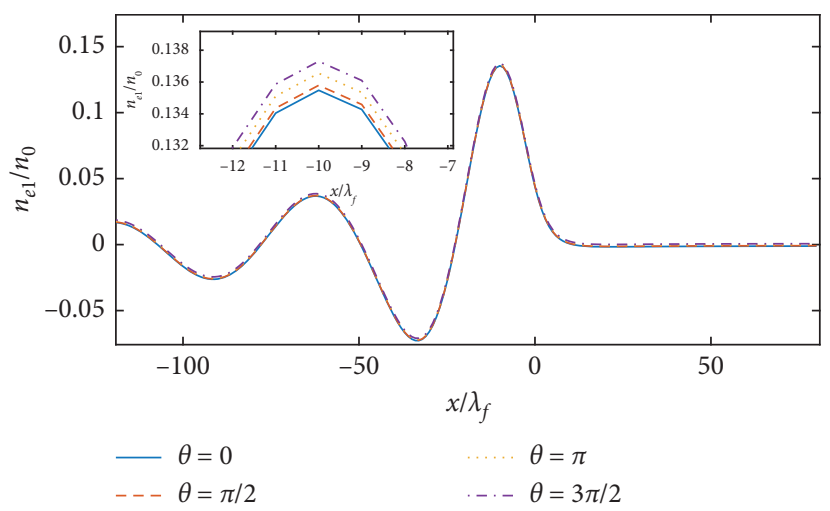

FIGURE 18: 2DQEG perturbation electron density. Here, $Z_{1}=1$, $r_{s}=1, v=2, \gamma=0.02, Z_{0}=9, \lambda=1.76 \times 10^{-4} \mathrm{~cm}$, and $\Phi_{0}=1 \times$ $10^{2} e / \lambda_{f}$.

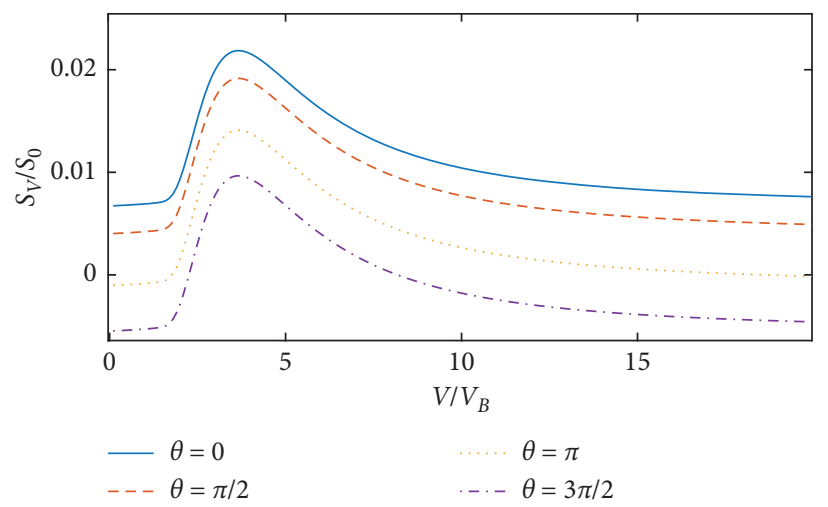

FIGURE 19: Stopping power of the incident charged particle. Here, $Z_{1}=1, r_{s}=1, \gamma=0.02, Z_{0}=9, \lambda=1.76 \times 10^{-4} \mathrm{~cm}$, and $\Phi_{0}=1 \times$ $10^{2} e / \lambda_{f}$

It is an exciting result that the average stopping power of the incident particles is negative in the region $v<2.3 v_{B}$ and $v>5.8 v_{B}$ when $\lambda=1.76 \times 10^{-4} \mathrm{~cm}$ and $\Phi_{0}=2 \times 10^{10} \mathrm{e} / \lambda_{f}$. The incident particles will gain energy and can be accelerated 


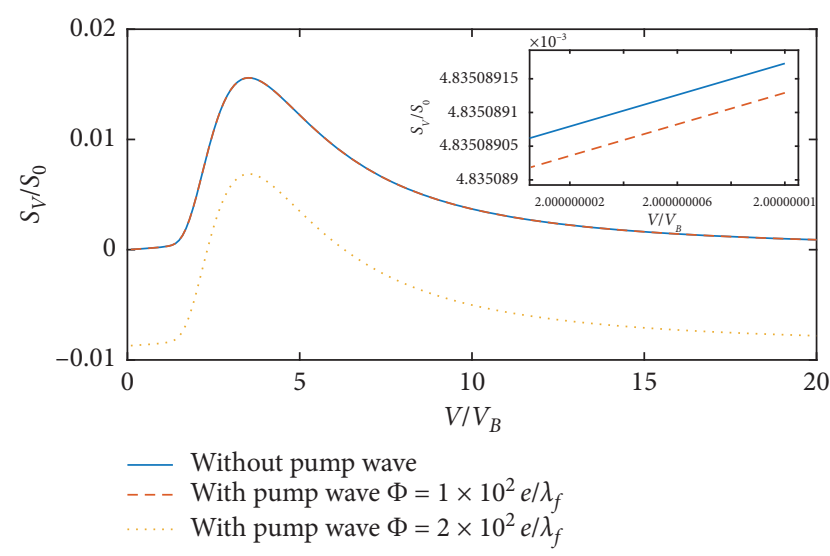

FIgURE 20: The average value of stopping power for time phase. Here, $Z_{1}=1, r_{s}=1, \gamma=0.02, Z_{0}=9$, and $\lambda=1.76 \times 10^{-4} \mathrm{~cm}$.

within the velocity range of $v<2.3 v_{B}$ and $v>5.8 v_{B}$. Although this result is not strictly obtained by using the linearized QHD due to the amplitude of pump wave which is too large, this could predict qualitatively that the time averaged stopping power has indeed been affected by the pump waves.

In conclusion, the time phase of pump wave $\theta$ can adjust the value of perturbation electron density effectively and stopping power, and the averaging of the stopping power with respect to the time phase $\theta$ also has been impacted. Therefore, one can see that the perturbation electron density and stopping power depend on $\lambda, \Phi_{0}$, and $\theta$, indicating that the pump wave using different parameters can modulate the perturbation electron density and stopping power of 2DQEG.

\section{Summary}

In this paper, considering the electron exchange-correlation potential $V_{x c 2 \mathrm{DH}}$ in QHD, we have calculated the perturbation electron density and stopping power with the modulation of pump wave for a charged particle with a constant distance and speed moving above 2DQEG. In our calculation, we compare the stopping power with and without $V_{x c 2 \mathrm{DH}}$ and show that it is necessary to consider $V_{x c 2 \mathrm{DH}}$ in 2DQEG. Then, we compare the perturbation electron density and stopping power with different wavelength $\lambda$, amplitude $\Phi_{0}$, and time phase $\theta$ of the pump wave and show that it is meaningful to investigate the modulation effect of the pump wave on the perturbation electron density and the stopping power. The wavelength $\lambda$, the amplitude $\Phi_{0}$, and time phase $\theta$ of the pump wave can effectively modulate the value of the perturbation electron density and stopping power. The temporal averaged stopping power with respect to the time phase $\theta$ also has been influenced by pump waves.

The possible experimental equipment to verify the prediction includes three components: pump wave, charged particle beam, and 2DQEG. The pump wave can be provided by laser or microwave device, the plasma wake field [52] can produce the charged particle beam, and 2DQEG has already been observed in experiments $[2,3]$. All of them can be realized with present technology. At present, the intensity of laser systems provided by the laboratory has arrived $10^{24} \sim 10^{26} \mathrm{Wcm}^{-2}$ [53]. Thus, the parameter of the pump wave considered here can be easily satisfied. We can verify the negative stopping power by measuring the velocity of the incident particle. For instance, charged particles move in the $x$ direction parallel to the 2DQEG plane, with a velocity of $v$ and a height of $z_{0}$ away from the 2DQEG, and the pump wave irradiates the 2DQEG along the $+x$ direction. The wavelength of pump wave is $\lambda=1.76 \times 10^{-4} \mathrm{~cm}$ and $\Phi_{0}=2 \times 10^{10} e / \lambda_{f}$. The density of two-dimensional electron gases is $n_{0}=5.69 \times 10^{15} \mathrm{~cm}^{-2}$. The velocity of incident particles is $v=2 v_{B}\left(v_{B}=2.18 \times 10^{8} \mathrm{~cm} / \mathrm{s}\right)$, and the distance between incident particles and two-dimensional electron gases is $z_{0}=9 \lambda_{f}=4.76 \times 10^{-8} \mathrm{~cm}$. The measured particles flying out the right side of the $2 \mathrm{DQEG}$ will gain energy from the 2DQEG and get accelerated.

It is appropriate to investigate the stopping power modulation by low-intensity pump waves of the charged particle moving above two-dimensional electron gases using the linearized QHD, but linearized QHD is not strictly applicable to high-intensity pump waves. In future work, we attempt to further study the effect of high-intensity pump waves on the stopping power with nonlinear QHD.

\section{Data Availability}

The data that support the findings of this study are available from the corresponding author upon reasonable request.

\section{Conflicts of Interest}

The authors declare that they have no conflicts of interest.

\section{Acknowledgments}

This work was supported by the National Natural Science Foundation of China (11975174 and 11775090), the National Magnetic Confinement Fusion Energy Research Project (2017YFE0301805 and 2017YFE0301803), and the Fundamental Research Funds for the Central Universities (WUT: 2020IB023 and 2018IB011).

\section{References}

[1] E. Abrahams, S. V. Kravchenko, and M. P. Sarachik, "Metallic behavior and related phenomena in two dimensions," Reviews of Modern Physics, vol. 73, no. 2, p. 251, 2001.

[2] X.-L. Qi and S.-C. Zhang, "Topological insulators and superconductors," Reviews of Modern Physics, vol. 83, no. 4, p. 1057, 2011.

[3] P. Di Pietro, M. Ortolani, O. Limaj et al., "Observation of Dirac plasmons in a topological insulator," Nature Nanotechnology, vol. 8, no. 8, p. 556, 2013.

[4] C.-Z. Li, Y.-H. Song, and Y.-N. Wang, "Electrical properties of epitaxial SrTiO3 tunnel barriers on (001) $\mathrm{Pt} / \mathrm{SrTiO} 3$ substrates," Physical Review A, vol. 79, Article ID 062903, 2009.

[5] C.-Z. Li, Y.-H. Song, and Y.-N. Wang, "Wake effects and stopping power for a charged particle moving above two-dimensional quantum electron gases," Physics Letters A, vol. 372, no. 24, p. $4500,2008$. 
[6] Y.-N. Wang and T.-C. Ma, "Consistent calculation of the stopping power for slow ions in two-dimensional electron gases," Physical Review A, vol. 55, no. 3, p. 2087, 1997.

[7] Z. L. Mišković, S. Segui, J. L. Gervasoni, and N. R. Arista, "Plasmons in graphene traversed by relativistic electronic beam with arbitrary trajectories," Physics Letters B, vol. 94, Article ID 125414, 2016.

[8] Z. L. Mišković, W.-K. Liu, F. Goodman, and Y.-N. Wang, "Spatial distribution of ion charges in fast, partially stripped clusters traversing solid targets," Nuclear Instruments and Methods in Physics Research Section B: Beam Interactions with Materials and Atoms, vol. 193, p. 221, 2002.

[9] G. Maynard and C. Deutsch, "Energy loss and straggling of ions with any velocity in dense plasmas at any temperature," Physical Review A, vol. 26, no. 1, p. 665, 1982.

[10] N. J. M. Horing, H. C. Tso, and G. Gumbs, "Fast-particle energy loss in the vicinity of a two-dimensional plasma," Physical Review B, vol. 36, no. 3, p. 1588, 1987.

[11] A. Bret and C. Deutsch, "Dielectric response function and stopping power of a two-dimensional electron gas," Physical Review E, vol. 48, no. 4, p. 2994, 1993.

[12] A. Bret and C. Deutsch, "Ion stopping in two-dimensional electron layers," Europhysics Letters (EPL), vol. 25, no. 4, p. 291, 1994.

[13] J. Lindhard and A. H. Rensen, "Relativistic theory of stopping for heavy ions," Physical Review A, vol. 53, no. 4, p. 2443, 1996.

[14] G. Zwicknagel, C. Toepffer, and P.-G. Reinhard, "Stopping of heavy ions in plasmas at strong coupling," Physics Reports, vol. 309, no. 3, p. 117, 1999.

[15] E. R. Custidiano and M. M. Jakas, "Classical-trajectory Monte Carlo calculations of the electronic stopping cross section for $\mathrm{keV}$ protons and antiprotons impinging on hydrogen atoms," Physical Review A, vol. 72, Article ID 022708, 2005.

[16] E. Zaremba, I. Nagy, and P. M. Echenique, "Nonlinear screening and stopping power in two-dimensional electron gases," Physical Review B, vol. 71, Article ID 125323, 2005.

[17] P. K. Patel, A. J. Mackinnon, M. H. Key et al., "Sequence dependent DNA-mediated conduction," Physical Review Letters, vol. 91, Article ID 125004, 2003.

[18] S. N. Chen, S. Atzeni, M. Gauthier et al., "Nuclear instruments and methods in physics research section A: accelerators, spectrometers, detectors and associated equipment," Proceedings of the First European Advanced Accelerator Concepts Workshop, vol. 740, p. 105, 2013.

[19] A. Zylstra, J. Frenje, P. Grabowski et al., "Non-equilibrium thermodynamics of gravitational screens," Physical Review Letters, vol. 114, Article ID 215002, 2015.

[20] J. Frenje, P. Grabowski, C. Li et al., "Negative tension branes as stable thin shell wormholes," Physical Review Letters, vol. 115, Article ID 205001, 2015.

[21] A. C. Hayes, G. Jungman, A. E. Schulz et al., "Reaction-inflight neutrons as a test of stopping power in degenerate plasmas," Physics of Plasmas, vol. 22, Article ID 082703, 2015.

[22] S. Y. Gus'kov, M. S. Solyanikova, and P. A. Korneev, "Specifics of powerful shock initialization by energetic ion beam," Plasma Physics and Controlled Fusion, vol. 61, Article ID 045006, 2019.

[23] J. Jacoby, D. H. H. Hoffmann, W. Laux et al., "Stopping of Heavy Ions in a Hydrogen Plasma," Physical Review Letters, vol. 74, no. 9, p. 1550, 1995.

[24] R. Cambridge, "LPB volume 36 issue 4 Cover and Front matter," Laser and Particle Beams, vol. 36, pp. 98-104, 2018.

[25] Z. D. Jieru Ren, "Pitfalls in identifying active catalyst species," Nature Communications, vol. 11, p. 5157, 2020.
[26] D. H. H. Hoffmann, N. A. Tahir, S. Udrea et al., "High energy density physics with heavy ion beams and related interaction phenomena," Contributions to Plasma Physics, vol. 50, no. 1, p. 7, 2010.

[27] D. H. H. Hoffmann, V. E. Fortov, M. Kuster et al., "High energy density physics generated by intense heavy ion beams," Astrophysics and Space Science, vol. 322, no. 1-4, p. 167, 2009.

[28] A. Frank, A. Blažević, P. L. Grande et al., "Phase field modeling of the evolution of partical interface shape distribution during coarsening," Physical Review E, vol. 81, Article ID 026401, 2010.

[29] E. Raphael and P.-G. d. Gennes, "Effective mass of a charged particle travelling above a dielectric fluid surface," Europhysics Letters (EPL), vol. 31, no. 5-6, p. 293, 1995.

[30] T. Lafleur and S. D. Baalrud, "Transverse force induced by a magnetized wake," Plasma Physics and Controlled Fusion, vol. 61, no. 12, Article ID 125004, 2019.

[31] T. Lafleur and S. D. Baalrud, "Friction in a strongly magnetized neutral plasma," Plasma Physics and Controlled Fusion, vol. 62, no. 9, Article ID 095003, 2020.

[32] Y. Zhang, W. Jiang, and L. Yi, "Stopping power of twodimensional spin quantum electron gases," Nuclear Instruments and Methods in Physics Research Section B: Beam Interactions with Materials and Atoms, vol. 349, p. 72, 2015.

[33] N. R. Arista, R. M. O. Galvão, and L. C. M. Miranda, "Influence of a strong laser field on the stopping power for charged test particles in nondegenerate plasmas," Journal of the Physical Society of Japan, vol. 59, p. 544, 1990.

[34] H. B. Nersisyan and E. A. Akopyan, "Stopping and acceleration effect of protons in a plasma in the presence of an intense radiation field," Physics Letters A, vol. 6, 1999.

[35] Z.-H. Hu, Y.-H. Song, Z. L. Mišković, and Y.-N. Wang, "Energy dissipation of ion beam in two-component plasma in the presence of laser irradiation," Laser and Particle Beams, vol. 29, no. 3, p. 299, 2011.

[36] Y. Zhang, Y.-H. Song, and Y.-N. Wang, "Nonlinear wake potential and stopping power for charged particles interacting with a one-dimensional electron gas," Physics of Plasmas, vol. 18, no. 11, Article ID 112705, 2011.

[37] Z.-H. Hu, Y.-H. Song, and Y.-N. Wang, "Critical properties of the S4 model for Sierpinski carpet," Physical Review E, vol. 85, Article ID 016402, 2012.

[38] Y. Zhang, Y.-H. Song, and Y.-N. Wang, "Simulations of interactions of high-energy proton beam with high dense matter based on two-dimensional quantum hydrodynamic model," Laser and Particle Beams, vol. 31, no. 2, p. 345, 2013.

[39] D. Wu, S. X. Luan, J. W. Wang et al., "Plasma detachment in linear devices," Plasma Physics and Controlled Fusion, vol. 59, Article ID 065004, 2017.

[40] Z. Mir, M. Shahid, M. Jamil, A. Rasheed, and A. Shahbaz, "Nonlinear beat excitation of low frequency wave in degenerate plasmas," Physics Plasmas, vol. 8, 2018.

[41] F. Stern and W. E. Howard, "Properties of semiconductor surface inversion layers in the electric quantum limit," Physical Review, vol. 163, no. 3, p. 816, 1967.

[42] Y. Zhang and W. Jiang, "Pseudomagnetic field modulation of stopping power for a charged particle moving above graphene," Physics of Plasmas, vol. 25, Article ID 072107, 2018.

[43] Y. Zhang, Y.-H. Song, Y.-T. Zhao, and Y.-N. Wang, "Twodimensional quantum hydrodynamic model for the heating of a solid target using a Gaussian cluster," Laser and Particle Beams, vol. 30, no. 4, pp. 671-677, 2012. 
[44] P. Kumar, S. Singh, and N. Ahmad, "Conversion efficiency of even harmonics of whistler pulse in quantum magnetoplasma," Laser and Particle Beams, vol. 37, no. 1, pp. 5-11, 2019.

[45] P. Kumar and N. Ahmad, "Surface plasma wave in spin-polarized semiconductor quantum plasma," Laser and Particle Beams, vol. 38, no. 2, pp. 159-164, 2020.

[46] L.-Y. Zhang, X.-Y. Zhao, X. Qi, W.-S. Duan, G. Q. Xiao, and L. Yang, "Collective effects on the wakefield and stopping power of an ion beam pulse in plasmas," Physics of Plasmas, vol. 22, Article ID 053109, 2015.

[47] F. Haas, "Kinetic theory derivation of exchange-correlation in quantum plasma hydrodynamics," Plasma Physics and Controlled Fusion, vol. 61, Article ID 044001, 2019.

[48] L. Pollack and J. P. Perdew, "Evaluating density functional performance for the quasi-two-dimensional electron gas," Journal of Physics: Condensed Matter, vol. 12, no. 7, pp. 1239-1252, 2000.

[49] F. Stern and S. Das Sarma, "Electron energy levels in GaAs-Ga1-xAlxAsheterojunctions," Physical Review B, vol. 30 , no. 2, p. $840,1984$.

[50] L. Brey, J. Dempsey, N. F. Johnson, and B. I. Halperin, "Infrared optical absorption in imperfect parabolic quantum wells," Physical Review B, vol. 42, no. 2, p. 1240, 1990.

[51] Y. Zhang, F. Zhai, and L. Yi, "Study of spin-polarized plasma driven by spin force in a two-dimensional quantum electron gas," Physics Letters A, vol. 380, no. 46, p. 3908, 2016.

[52] W. Yao, B. Li, L. Cao et al., "Generation of monoenergetic proton beams by a combined scheme with an overdense hydrocarbon target and an underdense plasma gas irradiated by ultra-intense laser pulse," Laser and Particle Beams, vol. 32, no. 4, p. 583, 2014.

[53] A. J. Kemp, F. Fiuza, A. Debayle et al., "Laser-plasma interactions for fast ignition," Nuclear Fusion, vol. 54, Article ID 054002, 2014. 